\title{
RMAIS: RFID-based Medication Adherence Intelligence System
}

\author{
Corey McCall, Student Member, IEEE, Branden Maynes, \\ Cliff C. Zou, Member, IEEE and Ning J. Zhang
}

\begin{abstract}
There has been compelling evidence that outpatients, especially those who are elderly or taking multiple complexly scheduled drugs, are not taking their medicines as directed, leading to unnecessary disease progression, complications, functional disabilities, lower quality of life, and even mortality. Existing technologies for monitoring and improving drug adherence are either costly or too complicated for general patients to use. In this paper, we introduce the detailed design and the complete prototype of a marketable Radio-Frequency Identification (RFID)-based Medication Adherence Intelligence System (RMAIS) that can be conveniently used at a residential home by ordinary patients. RMAIS is designed to maintain patients' independence and enable them to take multiple daily medicine dosages of the right amount at the right time. The system is patient-centered and user-friendly by reminding a patient of the prescribed time for medication and dispensing it in a fully automatic and fool-proof way. This is achieved mainly due to its novel design of a motorized rotation platform and the smooth integration of a scale, an RFID reader, and the rotation platform. In addition, this system has an Internet-based notification function that is used to alert the patient when it is time to take medicine as well as report deviations from the prescribed schedule to the primary care physicians or pharmacists.
\end{abstract}

\section{INTRODUCTION}

According to the national council report [1], "In the United States and around the world, there is compelling evidence that patients are not taking their medicines as prescribed, resulting in significant consequences." Medication noncompliance can result in unnecessary disease progression, complications, lower quality of life, and even mortality. As medical science has made possible new therapies and medicines to effectively treat more chronic or fatal diseases, medication schedules and conflicts between medicines have become more complicated and difficult for general patients to grasp. This problem is even worse for elderly patients who are forgetful or have dementia.

The growing need for in-home healthcare devices is best described in [4] as the population growth of retirement-age Americans is projected to overload the current healthcare system and inevitably cause it to fail in less than ten years.

Manuscript received April 1, 2010. This work was supported in part by the National Science Foundation under Grant CNS-0627318.

Corey McCall, Branden Maynes and Cliff C. Zou are with the School of Electrical Engineering and Computer Science, University of Central Florida, 4000 Central Florida Blvd., Orlando, FL 32816 USA (phone: 407823-5015; fax: 407-823-5835; e-mail: czou@eecs.ucf.edu).

Ning J. Zhang is with College of Health and Public Affairs, University of Central Florida, 4000 Central Florida Blvd., Orlando, FL 32816 USA (email: nizhang@mail.ucf.edu).
Other authors, such as in $[5][6][7][8]$, support the implementation of more in-home healthcare technology at the present time because it greatly increases the efficiency of caregivers and lowers healthcare cost. This cost not only includes money, but also the burden to caregivers, many of which are volunteering friends or family members.

For the above reasons, it is critical to develop an in-home healthcare device that can facilitate medication adherence. In this paper, we develop an inexpensive and marketable Radio-Frequency Identification (RFID)-based Medication Adherence Intelligence System (RMAIS) that will enable patients to follow prescribed medication schedules with minimal effort. Although some innovative technologies have been developed in recent years to allow us to monitor and improve drug adherence, such as [2][3], they are either too expensive to be adopted, or not convenient enough for patients to use. This is especially the case for elderly patients who are not familiar or comfortable with complex operations on high-tech devices.

RMAIS is built on a mature engineering technology -RFID. The system is practical and inexpensive to implement since it only requires each participating pharmacy to install a simple RFID writer (around \$130 USD) for generating RFID tags (currently around $\$ 0.20$ per tag) that can be attached to medicine bottles. It does not require any other alteration in the current manufacturing and pharmacy operating procedures. Just like a regular printed label on a medicine bottle, the data written to an RFID tag specifies the medicine name, schedule, dosage and special instructions.

We have actually developed a complete prototype of RMAIS, which will be introduced in detail in this paper. RMAIS has the following features:

- The system can alert patients by alarm or cell phone text-message when it is time to take medicine.

- Data on a medicine bottle's RFID tag enables the system to automatically read the medicine's information without requiring manual input from the patient or caregiver.

- With the built-in scale, the system can automatically tell if a patient has taken the right amount of dosage for each medicine.

- With the motorized rotation platform and each medicine bottle's RFID data feedback, the system can automatically rotate the correct medicine bottle in front of a patient for her to take - no need to decide which bottle to pick.

- With the built-in network interface card, the system can automatically alert a patient's healthcare provider 
or pharmacist (via email or text-message) of overdose or underdose incidents.

The rest of this paper is organized as follows. Section II introduces several closely related research projects. In Section III, we present the detailed system architecture and designs. In Section IV, we discuss how a patient operates the RMAIS device. Finally we conclude the paper in Section V.

\section{RELATED WORK}

The proposed RMAIS system is somewhat similar to the systems in [2][3][12]. INRange Systems Inc. developed an FDA-approved in-home medication dispensing box [2], which can automatically dispense the right amount of pills and alert the patient at the right time. However, this product has two major problems: 1). All pills need to be individually packed in special-made hard-paper board, which can only be done by medicine manufacturers (not by pharmacists). This greatly increases medication price and limits its adoption by the healthcare system. 2). It is only applicable for pill format medicine and cannot be used for liquid medicine.

The in-home medication system presented in [3][12] utilizes scale and RFID technology. However, it requires a patient to put all medicine bottles on top of a scale and measure all of the bottles' weight together, which makes the system not suitable for patients with many medicines and also requires an expensive high-capacity scale. In addition, it requires the patient to manually pick up the right bottle from the cluster of bottles on top of the scale, making the system inconvenient to use.

\section{RMAIS ARCHITECTURE AND PROTOTYPE DESIGN}

RMAIS is composed of five parts: RFID reader, scale, microcontroller, LCD panel, and motorized rotation platform. In order to manufacture the prototype, we used SolidWorks 3D CAD design software [9] to generate the detailed device model. Fig. 1 shows the assembly model of RMAIS generated in SolidWorks. In our prototype, all the transparent parts shown in Fig. 1 are plastic. Fig. 2 shows the bird's-eye view with detailed labeling of each component. Fig. 3 shows the photo of our final prototype.

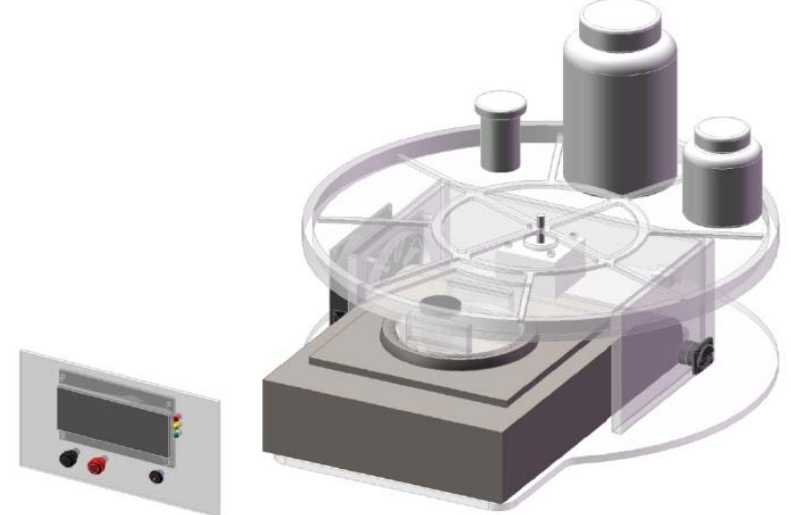

Figure 1: SolidWorks assembly model of the prototype RMAIS device (trimetric view)

\section{A. Scale}

Because we need to weigh medicine bottles to detect whether a patient has taken the correct number of pills, the scale needs to have an accuracy of around 1 milligram since some medicine pills are as small as just a few tens of milligrams. In addition, it needs to have a communication interface to receive control signals from the microcontroller and send weight measurements back to the microcontroller. For these reasons, the scale is the most expensive component in our prototype. We purchased the Acculab Vicon Portable Digital Balance VIC-303 and RS-232 interface kit. The combined cost is around \$350 USD.

In our tests, the integrated scale was able to flawlessly detect a single missing pill in a large bottle with as many as 250 other identical pills, as well as in a smaller bottle by itself. The smallest pill that we tested was $150 \mathrm{mg}$ and the largest was $1500 \mathrm{mg}$.

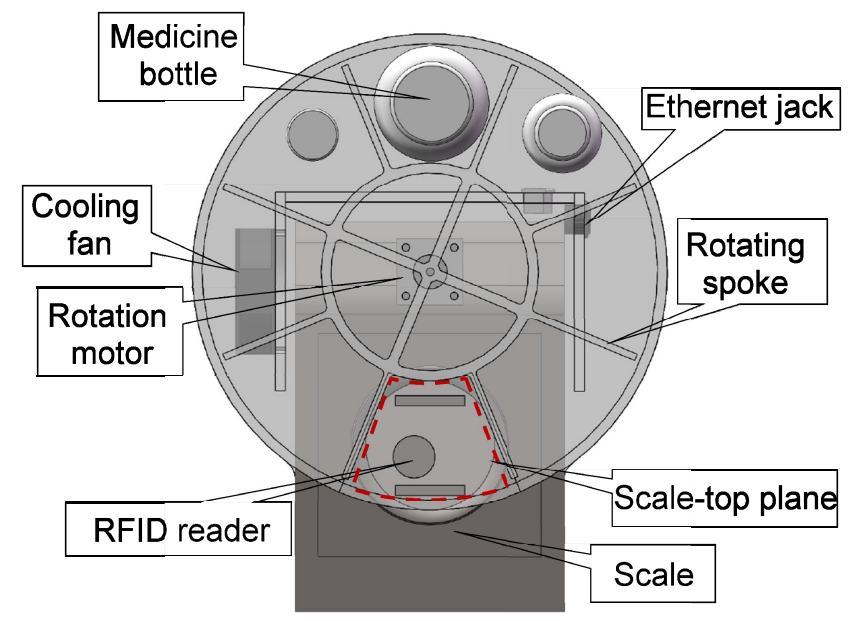

Figure 2: Bird's-eye view of the prototype RMAIS (not including the LCD display panel). The small microcontroller is behind the scale and under the rotation step motor.

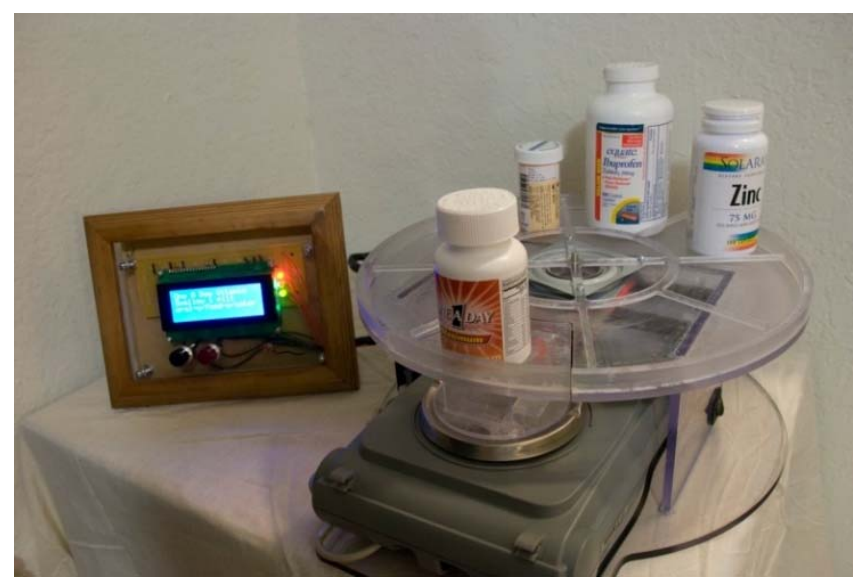

Figure 3: Picture of our final RMAIS prototype (the LCD panel displays the medicine name and dosage information of the bottle on the scale-top plane)

\section{B. Motorized Rotation Platform}

The most novel design of RMAIS is the motorized rotation platform. As illustrated in Fig. 1, 2, and 3, medicine bottles are placed on a round, stationary platform with a raised outer edge wall. A raised spoke is placed on top of the 
platform and fixed to a step motor that allows it to rotate. With this design, each medicine bottle is confined within a section defined by the spoke and edge wall. Medicines can then be easily pushed around by the rotating spoke, passing through the special section of the platform above the scale for weight measurement. We call this special section the "scale-top plane".

In order to weigh a medicine bottle, the scale-top plane is attached to the scale, while being barely separated from the remaining part of the platform (as shown in Fig. 4). In fact, when manufacturing the platform, we cut this section out of the larger round piece, grinded down the inside edges, and then attached it to the scale's measurement tray. We used two rectangular plastic parts to lift it up to the same height as the main platform. In this way, a bottle can be pushed by the rotating spoke and seamlessly slide from the main platform to the scale-top plane surface, and can then be weighed by the scale without any interference from the rest of the platform.

The step motor is controlled by the microcontroller and moves only a fraction of a degree at each step. Thus we can accurately control the rotating spoke to push a bottle to the scale-top plane to be weighted.

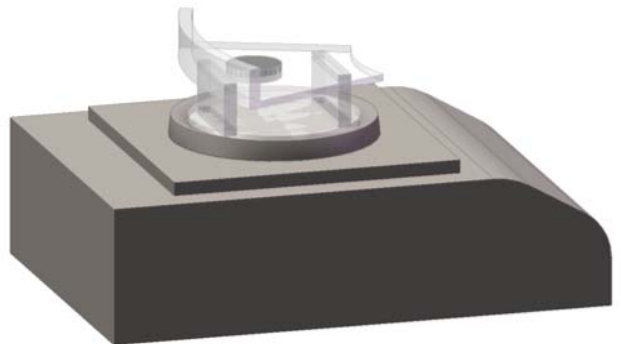

Figure 4: Model of the scale-top plane assembly fixed to the scale. When a medicine bottle is moved to this section of the platform, it can be weighed independently from the other bottles since the section is physically separated. The gray button pasted under the scale-top plane is the RFID reader.

\section{RFID Reader and Tags for Medicine Bottles}

There are many types of RFID tags available with different wireless transmission ranges, frequencies, sizes and costs. What we need are simple, low-cost passive RFID tags that can be attached to medicine bottles, and have a small data storage for storing medicine information. Since we do not need the tags to have a long data transmission range, the passive RFID tags can be purchased at a very cheap price. For our prototype, we purchased Texas Instruments (VA) 13.56MHz RFID Transponders (Tag RI-I17-112A-03), which cost around $\$ 1$ per tag. The cost is high because we only bought a dozen tags. In large quantities, each tag could be as low as 5 cents [10].

The tags we purchased have the shape and size of a US quarter coin, and are as thin as normal print paper. This enables us (or a pharmacist) to easily paste a tag onto the bottom of each medicine bottle.

For the RFID reader, as shown in Fig. 5, we purchased a coin-size $13.56 \mathrm{MHz}$ mini RFID reader manufactured by Skyetek Inc. (SkyeModule M1-Mini) that has a read range of around 3 to $4 \mathrm{~cm}$. It is compatible with the RFID tags and can be mounted to the undersurface of the scale-top plane (as shown in Fig. 2 and 4). With this setup, when a medicine bottle is pushed across the scale-top plane, there is less than $1 \mathrm{~cm}$ distance between the RFID reader and the tag on the bottom of the bottle. This allows the reader to read and only read the tag of the bottle on the scale-top plane without the need to consider the possible interference from the RFID tags of the other bottles on the platform.

We positioned the RFID reader such that it is able to read any size bottle pushed onto the platform by the rotating spoke. We incurred a problem when the user places a small medicine bottle in one of the inside corners of the scale top plane. Since the reader's integrated antenna is so small, it would not acknowledge the bottle's presence. Future development should improve the reader by using a slightly larger antenna.

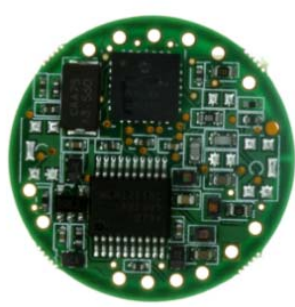

Figure 5: The coin-size mini RFID reader pasted on the bottom of the scaletop plane for reading the tag on each medicine bottle. This RFID reader is only $25.4 \mathrm{~mm}$ in diameter and $2.8 \mathrm{~mm}$ in height.

\section{Microcontroller}

The microcontroller we used in our prototype is the Arduino Mega [11], which is a microcontroller board based on the ATmega1280. It has all the digital/analog IO interfaces we need. Without any requirement on its computation speed, this microcontroller is small enough to fit in our prototype (behind the scale and under the rotation step motor), and it is cheap with a price of around $\$ 65$.

We also installed an Ethernet interface (NKC Ethernet Shield for Arduino Mega) to the microcontroller. This enables Internet access from the RMAIS system. With some simple networking programming, the system can send an alert email or a text message (such as to a cell phone) to its patient as a medication reminder, or to its patient's healthcare provider as a noncompliance alert. The Internet connectivity greatly enhances functionality and could provide any network related functions we can think of in future development.

\section{E. LCD Panel}

The last component of RMAIS is the LCD panel. It is used to display medicine information of the bottle on top of the scale-top plane. This is similar to what is printed on the paper label, including medicine name, dosage, special instructions, etc. It is also used to display system information such as alert messages and the current time. In our prototype, the LCD panel only costs around \$20.

\section{RMAIS PATIENT OPERATION}

In this section, we present how a patient operates the RMAIS device. 


\section{A. Initialization}

When a patient brings the RMAIS device home, it needs to be initialized and input all existing medicine information into the system.

The design of RMAIS makes the initialization a very simple step. Once the patient connects the device to a power and Ethernet line, it will automatically obtain an IP address from an Internet cable modem or wireless router in the same way as a normal computer. The microcontroller uses network time protocol (NTP) to connect to a predefined time server (there are many free time servers on the Internet) to automatically setup its clock time.

After the clock is set, the patient can put her medicine bottles onto the scale-top plane one at a time. Once a bottle is placed on the scale-top plane, RMAIS uses the RFID reader to automatically read the bottle's tag data, save the medicine information into memory, and then rotate the spoke to empty the scale-top plane for the next medicine. This user-friendly operation removes the burden of manually inputting all medicine information into the system.

When the patient purchases a new medicine, she simply places it onto the scale-top plane, and RMAIS will automatically read it into the system.

\section{B. Medication Administration}

When it is the scheduled time for the patient to take one or more medicines, RMAIS will generate an alarm sound (our prototype has a small buzzer installed). It can also be configured to send out a text-message through its Ethernet connection to the patient's cell phone or pager. In this way, the patient will surely get the reminder even if she is not close to the RMAIS device or has a hearing problem.

With the Internet support, RMAIS can ensure medicine adherence even when the patient is not at home. Before the patient leaves for a trip, she pushes an "out of home" button, then takes any number of medicine bottles off the device with her. When it is the scheduled time to take medicine, RMAIS will send a text-message to the patient's cell phone showing what medicine and what dosage she should take. After the patient comes back home and places those bottles back onto the device, RMAIS checks each bottle's weight to determine if the patient has taken the right amount of medicine during the trip.

When RMAIS sends out an alert to remind the patient to take medicine, it automatically activates the rotating spoke until the first medicine bottle for this time is pushed onto the scale-top plane. It then weighs the bottle and displays the medicine's name and dosage on the LCD panel. Once the patient takes some pills out of the bottle and puts it back onto the scale-top plane, the bottle is weighed again. Based on the difference between the two weight measurements and each pill's weight (pill weight is stored in the medicine's RFID tag), RMAIS can know if the patient has taken the right amount of medicine.

If the patient has not taken enough dosage of a medicine, RMAIS sounds its alarm and shows text reminding her to take the remaining dosage. If the patient takes too much dosage such that the overdose is dangerous, it will send a text-message to the patient's healthcare provider.

\section{Constant Medication Monitoring}

Since RMAIS will be used by elderly patients who may not be familiar with operating electronic equipment, it is likely that they may respond in an unexpected manner. For example, a user may take a medicine bottle from the platform before prompted to do so, or the user may replace a medicine bottle on another section of the platform other than the scale-top plane. The software on our prototype is designed to handle these unexpected inputs and changes.

Every time the user interacts with a medicine bottle, its weight is recorded and saved as its "most recent weight." The next time that medicine is to be taken, its weight is compared to its most recent weight, and if any pills are missing, the caregiver is alerted. RMAIS can also recognize foreign bottles on the platform by comparing each bottle's RFID tag against a list of known medicines as it passes across the scale-top plane. If the medicine is not recognized, it is automatically added to the system.

\section{CONCLUSION}

In this paper, we develop a practical medicine adherence intelligence system called RMAIS that can conveniently assist patients taking their medicines with the right dosage at the right time. We have developed a complete prototype that works as planned. The next step for us is to conduct a clinical trial, and push for commercialization of the system in order to make a real impact in improving the quality of healthcare and medication administration.

\section{REFERENCES}

[1] National Council on Patient Information and Education (2007) "Enhancing Prescription Medicine Adherence: A National Action Plan.”

http://www.talkaboutrx.org/documents/enhancing_prescription_medic ine_adherence.pdf

[2] EMMA in-home medication dispensing box. http://www.inrangesystems.com/

[3] Melody Moh, Loc Ho, Zachary Walker, Teng-Sheng Moh. "A prototype on RFID and sensor networks for elderly health care." In RFID Handbook: Applications, Technology, Security, and Privacy. Edited by Syed Ahson, Mohammad Ilyas, CRC; 1 edition (March 18, 2008).

[4] Ishman, E. "Inventing Wellness Systems for Aging in Place." IEEE Computer 37.5 (2004): 34-41.

[5] Boric-Lubecke, O. and V. M. Lubecke, "Wireless House Calls: Using Communications Technology for Health Care and Monitoring." IEEE Microwave Sept. 2002: 43-48.

[6] Fishkin, K. and M. Wang, A Flexible, Low-Overhead Ubiquitous System for Medication Monitoring. Rep. no. IRS-TR-03-011. Seattle, WA: Intel Corporation, 2003.

[7] Kenner, A. M. "Securing the Elderly Body: Dementia, Surveillance, and the Politics of "Aging in Place'" Surveillance \& Society 5.3 (2008): 252-69.

[8] Varshney, U. "Pervasive Healthcare and Wireless Health Monitoring." Mobile Networks and Applications 12 (2007): 113-27.

[9] SolidWorks 3D CAD Design Software. http://www.solidworks.com/

[10] Mark Roberti. "Tag Cost and ROI". http://www.rfidjournal.com/article/articleview/796/

[11] Arduino Mega. http://arduino.cc/en/Main/ArduinoBoardMega

[12] Fishkin, K. and M. Wang, "A Flexible, Low-Overhead Ubiquitous System for Medication Monitoring”. Rep. no. IRS-TR-03-011. Seattle, WA: Intel Corporation, 2003. 